\title{
Efeito do Symphytum officinale na consolidação de fraturas de rádio e ulna de ratos
}

\author{
Symphytum officinale effect on the consolidation of radio and ulna fractures in rats
}

Fabio Lucas Rodrigues'1, Rodrigo Luiz Vetorazzi', Cleber Furlan'

\section{Resumo}

Introdução: 0 estudo da consolidação óssea e dos diversos fatores capazes de estimular o processo de reparo ósseo envolve muitas pesquisas na literatura. Objetivo: testar o efeito do Symphytum officinale na consolidação de fraturas de rádio e ulna em ratos. Método: os autores avaliaram 34 ratos da linhagem Wistar, nos quais foi realizada fratura manual dos ossos do antebraço direito sob anestesia. Os ratos foram divididos aleatoriamente em dois grupos. 0 grupo experimento recebeu duas gotas da substância Symphytum officinale e o grupo controle recebeu duas gotas de soro fisiológico 0,9\%, administradas em ambos na frequência de três vezes ao dia pelo método da gavagem. Após o sacrifício, aos 14 e 28 dias de tratamento, foi realizada dissecção dos rádios e das ulnas. Os ossos foram radiografados e submetidos à leitura da densitometria óptica radiográfica. Resultados: foram observadas diferenças na formação do calo ósseo ulnar em relação ao radial, independentemente do grupo estudado na comparação entre o grupo controle e experimento. Conclusões: a formação do calo ósseo ulnar apresentou maior densidade óssea em relação ao calo radial, independentemente do grupo estudado, e a substância Symphytum officinale, em doses homeopáticas, não se mostrou estimulante da consolidação de fraturas em antebraços de ratos da raça Wistar.

Palavras-chave: Consolidação da fratura; confrei; ratos; calo ósseo.

\section{Abstract}

Introduction: there are several studies in medical literature covering the subject of bones consolidation, as well as the several stimulating factors related to it. Objective: to test the effect of Symphytum officinale in bone consolidation of radio and ulna fractures in rats. Method: the authors describe a survey conducted over a sample of 34 rats of Wistar lineage, randomly divided in two different groups, on which a manual procedure to cause the fractures in the bones of the right forearm was conducted with anesthesia. The rats of the experimental group were given two drops of a substance known as Symphytum officinale and the rats of the control group were given two drops of physiologic saline, $0.9 \%$. For those two groups, the drugs were given three times per day by gavage. After the sacrifice of the rats, a series of dissections of the radio and the ulna bones was conducted. All bones were submitted to X-rays and to optical densitometry radiographic devices. Results: a difference in the bone callus formation of ulna was observed when compared to the bone callus formation of radio, regardless of the groups. Conclusions: the bone callus formation of the ulna presented more bone density in relation to radio callus, independently to the studied group and Symphytum officinale, in homeopathic doses, did not stimulate the bone fractures consolidation in forearms of Wistar rats lineage.

Keywords: Fracture healing; comfrey; rats; bony callus.

Recebido: 26/6/2009

Modificado: 26/9/2009

Aprovado: $18 / 1 / 2010$

Trabalho realizado na Faculdade de Medicina do ABC (FMABC), Santo André (SP), Brasil.

Disciplina de Ortopedia e Traumatologia da Faculdade de Medicina do ABC (FMABC). Santo André (SP), Brasil

Endereço para correspondência: Fábio Lucas Rodrigues - Avenida Lauro Gomes, 2000 - Vila Sacadura Cabral - CEP 09060-870 - Santo André (SP) -

E-mail: fabiolucas@gmail.com 


\section{Introdução}

A consolidação óssea é assunto de grande interesse científico. Na prática médica, existe um crescente número de cirurgias ortopédicas com utilização de implantes para fixação interna de fraturas, alcançando a marca de dois milhões ao ano nos Estados Unidos' 1 .

O modelo experimental em ratos é de grande valia para sua realização no que se refere à reprodutibilidade e padronização da fratura a ser pesquisada ${ }^{2}$.

É consenso que vários fatores atuam no processo de resposta do osso ao trauma, incluindo o uso de fármacos ${ }^{3,4}$. Os corticosteroides, anticoagulantes, tiouracil e as vitaminas A e D, em altas doses, são destacados como contribuintes para o retardo da consolidação. Esteroides anabolizantes, hialuronidase, sulfato de condroitina, hormônio do crescimento, insulina, calcitonina, hormônios tiroidianos, vitaminas A e D em doses padrão ${ }^{3}$ e medicamentos homeopáticos, como o Symphytum officinale, agem estimulando a consolidação de fraturas ósseas ${ }^{5-7}$.

A Homeopatia, do grego homeos pathos, que significa "semelhante à doença”, e que foi desenvolvida no século 18 pelo médico alemão Frederico Samuel Hahnemann, fundamenta-se no princípio básico da "Lei dos Semelhantes". Toda substância capaz de provocar um quadro mórbido pode ser usada como medicamento para tratar um indivíduo doente que tenha um quadro semelhante ${ }^{5,7}$. Portanto, nessas condições, para ter efeito medicamentoso, uma substância deve ser diluída e dinamizada. Para obtenção da dinamização, normalmente os farmacêuticos utilizam, entre outras técnicas, as chamadas Centesimal Hahnemanniana $(\mathrm{CH})$ e Fluxo Contínuo (FC), que são as mais comuns?

O Symphytum officinale é uma planta da família Borraginácea, popularmente conhecida como Confreie ${ }^{5,6,8}$, contendo em sua composição altas doses de alcaloides pirrolizidínicos ${ }^{5,8}$, além de alantoína, tanino e esteroides ${ }^{5}$.

Essa substância, quando usada na sua forma natural e não diluída, provoca dores ósseas do periósteo e das articulações e retarda a consolidação óssea, aumentando a sensibilidade em relação à dor. Por isso, é denominada em homeopatia como medicamento "específico ortopédico" sendo indicada após traumatismo e fratura, pois diminui a dor e estimula a osteogênese ${ }^{7}$.

A Medicina Veterinária utiliza-se dos mesmos princípios e medicamentos usados em humanos ${ }^{9,10}$. Efeitos nocivos para os animais, como a hepatotoxicidade, são relatados na literatura apenas quanto ao uso das raízes e folhas verdes do Confrei na dieta dos ratos ${ }^{8}$. Na Alemanha, produtos derivados do Confrei são utilizados desde 1920 para fins medicinais ${ }^{6}$.

Dessa maneira, o objetivo é verificar o efeito estimulante do Symphytum officinale na consolidação óssea e avaliar a evolução natural da consolidação de fraturas de rádio e ulna em ratos.

\section{Método}

Foram utilizados 34 rattus novergicus albinus, da linhagem Wistar, machos, adultos, pesando $274 \pm 46 \mathrm{~g}$, mantidos em ambiente controlado, recebendo água e ração padrão ad libitum.
Os ratos foram anestesiados pela injeção intraperitoneal de solução composta de cetamina, na dose de $40 \mathrm{mg} / \mathrm{kg}$, e de xilazina, na dose de $5 \mathrm{mg} / \mathrm{kg}$ de peso corporal, diluídas em $1 \mathrm{~mL}$ de solução salina. Após anestesia adequada, foi provocada, manualmente, por um único pesquisador, fratura do antebraço direito dos animais, na região diafisária, utilizando-se três pontos de apoio. As fraturas não foram imobilizadas e os animais permaneceram sem restrições motoras dentro de suas gaiolas.

Após a fratura, os animais foram aleatoriamente divididos em dois grupos: controle e experimento. O grupo controle constituiu-se de 17 ratos, aos quais foram administradas duas gotas de solução fisiológica a $0,9 \%$, via oral, pelo método da gavagem, três vezes ao dia. O grupo experimento foi constituído por 17 animais, os quais receberam duas gotas de Symphytum officinale na potência de 6CH, pelo mesmo método e frequência do grupo anterior. Os ratos foram medicados do dia da fratura até a eutanásia. Esse procedimento realizou-se em dois momentos distintos, que subdividiram cada grupo em dois: após duas semanas do início do experimento (14 dias), em nove animais de cada grupo e, no final da quarta semana (28 dias), em oito animais, também de ambos os grupos.

A eutanásia dos animais ocorreu com administração de dose anestésica de barbitúrico, finalizando com dose letal de cloreto de potássio. As patas dianteiras direitas foram dissecadas, retirando-se todo invólucro de partes moles do rádio e da ulna.

Os antebraços foram submetidos à avaliação da densitometria óptica radiográfica (DOR), e o avaliador não sabia qual grupo estava examinando.

Os ossos de cada grupo (controle e experimento, com 14 e 28 dias), já dissecados, foram dispostos em posição anteroposterior em um chassi radiográfico, ao redor de uma escala de alumínio padrão, e numerados na imagem radiográfica (Figura 1). Esse procedimento foi realizado separadamente para cada grupo, totalizando-se quatro radiografias. Estas foram realizadas no mesmo aparelho, à mesma distância da ampola de raios X (um metro), pelo mesmo técnico em Radiologia e com a mesma técnica radiográfica (42 kv $\mathrm{x} 1,25 \mathrm{mAs})$.

As radiografias foram digitalizadas e analisadas por software de processamento e análise de imagem ImageLab. No total, realizaram-se três análises do rádio e da ulna separados, e a média foi utilizada para obtenção dos resultados.

Estatisticamente, os valores médios das leituras foram submetidos ao teste de Mann-Whitney, para comparação entre os dois grupos formados, e ao teste dos Postos Sinalizados de Wilcoxon, para comparação entre os dois momentos de observação. Adotamos nível de significância de $5 \%(\alpha=0,05)$, assinalando com asterisco $\left(^{*}\right)$ os valores significantes $(\mathrm{p}<0,05)$.

Esta pesquisa foi aprovada pela Comissão de Ética do Instituto de Ciências Avançadas e Otorrinolaringologia (ICAO) de São Paulo (SP), sob o processo 018/2006. 


\section{Resultados e discussão}

Na Tabela I, encontram-se os resultados referentes à comparação da média da densidade do calo ósseo formado no grupo experimento em relação ao controle, em que não se encontra diferença significativa entre esses grupos.

Nas Tabelas II e III observam-se as médias da densidade do calo ósseo ulnar e radial, comparadas entre os dois momentos da observação $\left(14^{\circ}\right.$ e $28^{\circ}$ dias), dos grupos experimento e controle, respectivamente. Em ambos os grupos, observamos maior densidade na ulna com 28 dias, quando comparada com 14 dias, fato que não se repetiu no rádio.

O ortopedista depara-se cada vez mais frequentemente, em sua prática diária, com fraturas consequentes de traumas de alta energia, como automobilísticos e por projétil de arma de fogo. Essas lesões têm maior dificuldade de consolidação, devido ao comprometimento vascular dos fragmentos ósseos. Pesquisas sobre substâncias ou métodos que estimulem a consolidação são usuais e importantes.

Apesar da extensa publicação experimental sobre consolidação das fraturas, não foi encontrada produção intelectual avaliando exclusivamente o uso do Symphytum officinale e sua relação com o estímulo da consolidação das fraturas, embora essa citação seja dada como verdadeira nos livros-texto homeopáticos?

Para tal estudo, utilizaram-se ratos Wistar (rattus novergicus albinus), machos, adultos, determinados pela sua grande ocorrência na literatura ${ }^{2,3,11-15}$ e pela facilidade de avaliação em quantidade, baixo custo relativo, facilidade no manuseio e possibilidade de simular, em seus ossos, fraturas de ossos humanos ${ }^{11}$.

Embora sejam frequentes os estudos com a utilização das fraturas nos membros inferiores dos ratos ${ }^{2-4,12-15}$, preferimos realizá-las no antebraço, fato não encontrado previamente na literatura. $\mathrm{O}$ menor apoio desse membro na marcha, teoricamente, em caso de fratura, resulta em diminuição da dor e da deformidade. Outra vantagem é a possibilidade de avaliar a consolidação de dois ossos longos num mesmo experimento.

A fratura fechada do rádio e da ulna pela técnica manual dos três pontos de apoio foi escolhida devido à fácil realização, reprodutibilidade e pela obtenção de traço transverso. A fratura produzida pelo método invasivo (osteotomia cruenta) tem complicações relatadas na literatura: aumento do risco de infecção, deiscência na sutura e maior índice de lesão em tecidos moles ${ }^{2,3,11}$.

Optou-se pela não fixação das fraturas com fio de Kirschner, baseando-se na ocorrência de diversas complicações, como infecção, mortes per-operatórias, cominuição da fratura na tentativa de passagem do fio, migração do fio e influência do material de síntese na formação do calo ${ }^{2,11-14}$.

A estabilização das fraturas por métodos não invasivos, como gesso ou bandagens, também não foi realizada devido à ocorrência de síndrome compartimental e destruição da imobilização pelos próprios animais ${ }^{2}, 12,13$. A ampla descrição de modelos que não empregam imobilização e a observação feita por diversos pesquisadores de que a con-

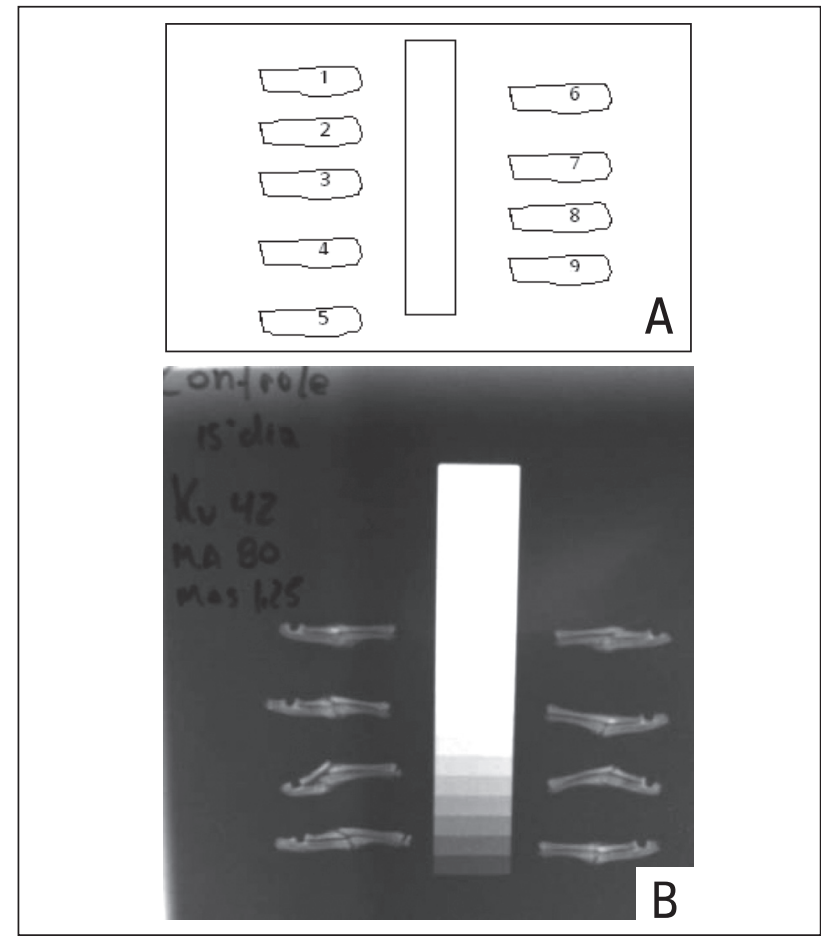

Figura 1 - (A) Esquema da disposição das peças na avaliação radiográfica; (B) Fotografia da radiografia obtida nos espécimes estudados.

Tabela I - Comparação dos grupos experimento e controle com o resultado do teste de Mann-Whitney

\begin{tabular}{lccccc}
\hline Variável & Grupo & $\mathrm{n}$ & Média & Desvio padrão & Significância $(\mathrm{p})$ \\
\hline \multirow{2}{*}{ r14 } & Experimento & 9 & 2,71 & 0,33 & \multirow{2}{*}{0,565} \\
& Controle & 9 & 2,54 & 0,50 & \\
\multirow{2}{*}{ u14 } & Experimento & 9 & 2,69 & 0,25 & 0,930 \\
& Controle & 9 & 2,73 & 0,31 & \\
\multirow{2}{*}{ r28 } & Experimento & 7 & 2,43 & 0,17 & \multirow{2}{*}{0,817} \\
& Controle & 8 & 2,50 & 0,28 & \\
\multirow{2}{*}{ u28 } & Experimento & 7 & 2,23 & 0,16 & \multirow{2}{*}{0,183} \\
& Controle & 8 & 2,09 & 0,12 & \\
\hline
\end{tabular}

r14: rádio após 14 dias; r28: rádio após 28 dias; u14: ulna após 14 dias; u28: ulna após 28 dias; n: número de animais; p: significância.

Tabela II - Grupo Experimento com o resultado do teste dos postos sinalizados de Wilcoxon

\begin{tabular}{lcccc}
\hline Par de variáveis & Média & $\mathrm{n}$ & Desvio padrão & Significância $(\mathrm{p})$ \\
\hline r14 & 2,71 & 7 & 0,34 & 0,176 \\
r28 & 2,43 & 7 & 0,17 & $0,018^{*}$ \\
u14 & 2,76 & 7 & 0,23 & \\
u28 & 2,23 & 7 & 0,16 & \\
\hline
\end{tabular}

r14: rádio após 14 dias; r28: rádio após 28 dias; u14: ulna após14 dias; u28: ulna após 28 dias; $n$ : número de animais; p: significância.

Tabela III - Grupo controle com o resultado do teste dos postos sinalizados de Wilcoxon

\begin{tabular}{lcccc}
\hline Par de variáveis & Média & $\mathrm{n}$ & Desvio padrão & Significância $(\mathrm{p})$ \\
\hline r14 & 2,48 & 8 & 0,50 & 0,779 \\
r28 & 2,50 & 8 & 0,28 & \\
u14 & 2,74 & 8 & 0,33 & $0,012^{*}$ \\
u28 & 2,09 & 8 & 0,12 & \\
\hline
\end{tabular}

r14: rádio após 14 dias; r28: rádio após 28 dias; u14: ulna após 14 dias; u28: ulna após 28 dias; n: número de animais; p: significância. 
solidação óssea é obtida sem uso de qualquer estabilização, abonam a opção dos autores pelo tratamento supracitado ${ }^{2,14-16}$.

A potência da substância, a dose administrada e a frequência foram baseadas em dados da literatura ${ }^{5}$.

A densitometria óptica é um dos métodos utilizados para medir a densidade mineral dos ossos, por meio de imagens radiográficas digitalizadas, comparando os tons de cinza dos calos formados com os tons de uma escala de alumínio, fornecendo a medida em milímetros de alumínio (mmAl), além de apresentar alta sensibilidade, reprodutibilidade e baixo custo ${ }^{17,18}$. Utilizamos essa técnica por ser objetiva para a obtenção dos resultados, diferindo de outros estudos que empregam metodologia subjetiva, como avaliação visual de radiografias².

Desconsideramos duas amostras do grupo controle com 28 dias de seguimento, devido à sobreposição das extremidades fraturadas do rádio que poderiam levar a um erro de leitura de densitometria óptica.

O resultado estatístico da DOR nos grupos controle e experimento apresentaram diferenças não significantes quanto à densidade do calo ósseo formado, demonstrando que a substância pesquisada não estimula a consolidação das fraturas nos antebraços dos ratos (Tabela I). Acreditamos que novos estudos com medicamentos homeopáticos devam ser realizados, com intuito de comprovar a impressão clínica - e dos livros-texto - de que a filosofia homeopática melhora a dor e estimula a consolidação em fraturas ósseas.

Em relação à verificação de possíveis diferenças entre os dois momentos da observação (14 e $28^{\circ}$ dias), constatamos significância estatística $(\mathrm{p}<0,05)$ na avaliação da densidade do calo ósseo ulnar aos 28 dias, tanto no grupo controle quanto no experimento, quando comparados aos valores encontrados aos 14 dias. A mesma avaliação foi realizada no rádio, porém não encontramos diferença estatisticamente significante (Tabelas II e III) que mostre a tendência desse osso evoluir para pseudartrose.

Assim, concluímos que a formação do calo ósseo ulnar apresenta maior densidade óssea na evolução da consolidação - diferente do rádio, que não evoluiu, independentemente do grupo estudado - e que a substância Symphytum officinale, em doses homeopáticas, não se mostrou estimulante da consolidação de fraturas em antebraços de ratos da raça Wistar.

\section{Agradecimentos}

Agradecemos ao professor Doutor Carlo Milani e à Doutora Caterina Muremoto pelo apoio à realização desta pesquisa.

\section{Referências}

1. Darouiche RO. Treatment of infections associated with surgical implants. N Engl J Med. 2004;350(14):1422-9.

2. Castro PCF, Hoshino A, Brito RB, Dias Júnior LB, Brito JAF, Barros RSM et al. Estudo do processo de consolidação óssea em ratos tratados com acetaminofen: avaliações radiográfica e histológica. Rev. bras. ortop. 2005;40(10):614-20.

3. Giordano M, Tamanini A, Giordano-Neto V, Apfel MIR, Elias N, Graça R et al. Influência da enoxaparina na consolidação das fraturas: estudo experimental em ratos. Rev. bras. ortop. 1999;34(3):208-13.

4. Beck A, Krischak G, Sorg T, Augat P, Farker K, Merkel U et al. Influence of diclofenac (group of nonsteroidal anti-inflammatory drugs) on fracture healing. Arch Orthop Trauma Surg. 2003;123(7):327-32.

5. Balducci-Roslindo E, Silvério KG, Malagoli DM. Processo de reparo em feridas de extração dentária em camundongos tratados com o complexo Symphytum officinale e Calendula officinalis. Rev Odontol Univ São Paulo. 1999;13(2):181-7.

6. Koll R, Buhr M, Dieter R, Pabst H, Predel HG, Petrowicz 0 et al. Efficacy and tolerance of a comfrey root extract (Extr. Rad. Symphyti) in the treatment of ankle distorsions: results of a multicenter, randomized, placebo-controlled double-blind study. Phytomedicine. 2004;11(6):470-7.

7. Cairo N. Guia de medicina homeopática. 22ª ed. Teixeira; São Paulo: 1986.

8. Hirono I, Mori H, Haga M. Carcinogenic activity of Symphytum officinale. J. Natl Cancer Inst. 1978;61 (3):865-9.

9. Wolff HG. Tratando o gato pela homeopatia. São Paulo: Andrei;1986.

10. Wolff HG. Tratando o cão pela homeopatia. São Paulo: Andrei; 1986
11. Jackson RW, Reed CA, Israel JA, Abou-Keer FK, Garside H. Production of a standard experimental fracture. Can J Surg. 1970;13(4):415-20.

12. Camargo-Júnior JNJ. Estudo experimental comparativo da morfologia e da dinâmica da população celular de reparação de fraturas de ossos longos tratadas com e sem osteossíntese intramedular. Tese (Doutorado) - USP São Paulo: 1972

13. Utvåg SE, Grundnes 0 , Rindal DB, Reikerås 0 . Influence of extensive muscle injury on fracture healing in rat tibia. J Orthop Trauma. 2003;17(6):430-5.

14. Guarniero R, Cinagava MY, Santana PJ, Batista MA, Oliveira LAA, Rodrigues CJ et al. Influência do componente proteico na consolidação de fraturas: trabalho experimental em ratos. Acta ortop. bras. 2003;11(4);206-10.

15. Giordano V, Knackfuss IG, Gomes RC, Giordano M, Mendonça RG, Coutynho F. Influência do laser de baixa energia no processo de consolidação de fratura de tíbia: estudo experimental em ratos. Rev. bras ortop. 2001; 36(5):174-8

16. Oztuna V, Ersöz G, Ayan I, Eskandari MM, Uğuz K, Kuyurtar F. Head injuryassociated bone fractures induce bacterial translocation: an experimenta study. J Orthop Trauma. 2004;18(2):92-5.

17. Muramoto C. Estabelecimento de valores de densidade mineral óssea (DMO) das regiões metafisária e diafisária do rádio em cães da raça Poodle por meio da densitometria óptica radiográfica. Mestrado (Dissertação) Universidade de São Paulo, São Paulo, 2003.

18. Talbott SM, Cifuentes M, Dunn MG, Shapses SA. Energy restriction reduces bone density and biomechanical properties in aged female rats. J Nutr. 2001;131(9):2382-7. 